Journal Club

Editor's Note: These short reviews of recent JNeurosci articles, written exclusively by students or postdoctoral fellows, summarize the important findings of the paper and provide additional insight and commentary. If the authors of the highlighted article have written a response to the Journal Club, the response can be found by viewing the Journal Club at www.jneurosci.org. For more information on the format, review process, and purpose of Journal Club articles, please see https://www.jneurosci.org/content/ jneurosci-journal-club.

\title{
Dissociated Neural Mechanisms of Target and Distractor Processing Facilitated by Expectations
}

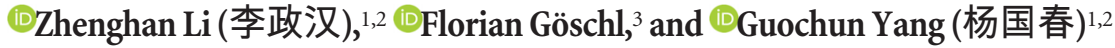 \\ ${ }^{1}$ CAS Key Laboratory of Behavioral Science, Institute of Psychology, Beijing 100101, China, ${ }^{2}$ Department of Psychology, University of Chinese Academy of \\ Sciences, Beijing 100101, China, and ${ }^{3}$ Department of Neurophysiology and Pathophysiology, University Medical Center Hamburg-Eppendorf, Hamburg \\ 20246, Germany
}

Review of van Moorselaar and Slagter

Our sensory systems constantly receive a tremendous amount of information, but only information that is attended can be well processed, because perceptual capacity is limited (Lavie, 2005). It has been suggested that selective attention upregulates task-relevant stimulus processing (target facilitation) and downregulates task-irrelevant stimulus processing (distractor inhibition; Baluch and Itti, 2011; Noonan et al., 2018). This attentional regulation has been shown to be influenced by expectations. For instance, in visual search, attention is biased toward spatial locations with higher probabilities of targets and away from locations with higher probabilities of distractors (Ferrante et al., 2018).

Although expectations can promote both target facilitation and distractor suppression, the underlying neural mechanisms appear to be different. Previous studies have demonstrated that expectations can act to enhance processing of tar-

Received Oct. 23, 2019; revised Jan. 15, 2020; accepted Jan. 18, 2020.

This work was supported by the National Natural Science Foundation of China (NSFC) and the German Research Foundation (DFG; Grants NSFC 61621136008 and DFG TRR-169/B4/A5/C1), and the China Postdoctoral Science Foundation (2019M650884). We thank Dr. Xun Liu, Dr. Jie Yuan, and Dr. Qing He for comments on the paper.

The authors declare no competing financial interests.

Correspondence should be addressed to Guochun Yang at yanggc@psych.ac.cn.

https://doi.org/10.1523/JNEUROSCI.2562-19.2020

Copyright $\odot 2020$ the authors gets even before the targets appear (for review, see Grothe, 2018). However, how processing of distractors is downregulated by expectations continues to be debated (Noonan et al., 2018). The dominant view holds that distractor processing is actively inhibited through a mechanism involving increased prestimulus oscillatory activity in the alpha frequency band $(8-12 \mathrm{~Hz}$; Jensen and Mazaheri, 2010). But two alternative mechanisms of distractor inhibition are also possible. One possible mechanism is secondary inhibition, in which deploying less attention to distractors results from actively allocating more attention to task-relevant stimuli. The other possible mechanism is expectationdependent suppression resulting from predictive coding in which both the expected target and distractor are suppressed, but the target is selectively released from suppression by top-down attentional control (Summerfield and Egner, 2009). Thus, how expectations downregulate distractor processing at the neural level remains an open question.

In a recent study, van Moorselaar and Slagter (2019) address this question in two behavioral experiments and an EEG experiment. Three different groups of participants performed a visual search task in which two Gabor patches were presented inside two of several placeholder circles on a visual display. The target patch was tilted either $45^{\circ}$ or $135^{\circ}$ while the distrac- tor Gabor was oriented either vertically or horizontally. Participants were instructed to indicate the orientation (left or right tilt) of the target Gabor via keyboard response as quickly and accurately as possible. The behavioral results indicated that repeatedly presenting the distractor at the same placeholder location (allowing participants to predict where it would appear) shortened the reaction time and increased the search efficiency. Importantly, the authors also manipulated the search display set size (i.e., each sequence contained either 4 or 8 placeholder circles) and found that the set size did not modulate learning efficiency in either the distractor-repeat condition or the targetonly variable condition (in which no distractor was present). This finding excluded the possibility that the suppression of distractor processing in the distractor-repeat condition reflects facilitated processing at locations never containing a distractor.

Based on the behavioral findings, the EEG experiment was designed to characterize the neural mechanisms underlying learning-related distractor suppression. With always six search placeholder circles and a four-trial sequence, the EEG experiment replicated the basic behavioral pattern that distractor learning reliably increased the search speed. Various methods were applied for the EEG data analy- 
ses. First, an inverted encoding model (IEM; Brouwer and Heeger, 2009) analysis allowed the authors to reconstruct activities of the neuronal populations tuned to distractor or target locations, with greater slope of a "channel tuning function" (CTF) indicating greater location selectivity. The CTF slope calculated based on the distribution of alpha power increased when the target was repeated, which revealed that learned expectations about the target location enhanced the spatial tuning of neuronal populations responsive to the target location both before and after the target was presented. However, no increase of the CTF slope was observed in the distractor-repeat condition, suggesting that the observed reduction in distractor interference at the behavioral level was not mediated by changes in the neural representation of the distractor location. Next, event-related potential (ERP) analyses were applied to examine the effect of learning on distractor and target processing after stimulus presentation. This analysis revealed that target repetition resulted in an earlier offset of the N2pc (an early ERP component reflecting attentional selection), whereas distractor repetition selectively reduced the amplitude of the Pd component (a neural marker of active distractor inhibition). Finally, multivariate decoding analysis was conducted to examine whether the neural signal comprised locationspecific information that was not captured by the IEM. This analysis showed that discrimination of the target location occurred only after onset of the search display. The decoding results converged with the ERP findings insofar as target repetition was associated with a shorter duration of the target location representation within the time window of the N2pc component.

The study by van Moorselaar and Slagter (2019) demonstrates that expectation-based target facilitation and distractor suppression rely on distinct neural mechanisms. The expectation about target location increases anticipatory alpha activity, whereas the expectation about distractor location only modulates the processing of the distractor after its appearance. Surprisingly, as indicated by the reduced $\mathrm{Pd}$ amplitude, active inhibition of distractor processing diminishes when expectations are formed, as if the brain learns that the distractor can be safely ignored. These results resonate with other recent studies (Noonan et al., 2016; Poch et al., 2018; for review, see Foster and Awh, 2019), but challenge the view that distractor filtering is implemented through anticipatory alpha activity (Jensen and Mazaheri, 2010; Payne et al., 2013).

Van Moorselaar and Slagter (2019) argue that continuous active inhibition of distractors would be an inefficient strategy of the brain to deal with irrelevant information and instead they interpret their findings in a predictive coding framework (Friston, 2009; Arnal and Giraud, 2012). According to this framework, expected stimuli evoke reduced neural activities; this has been observed in several studies (for review, see de Lange et al., 2018). When a distractor can be reliably predicted and thus the need for top-down modulation is reduced, the corresponding neural activity is almost silent, as indexed by the absence of the Pd component in the study by van Moorselaar and Slagter (2019). However, these findings suggest that learned expectations regulate distractor processing rather than refute the idea that active inhibition occurs. Indeed, expectation-dependent suppression and active inhibition are likely complementary mechanisms of distractor processing, and which of these two prevails might depend on the specific requirements of the situation (Noonan et al., 2018).

The findings presented by van Moorselaar and Slagter (2019) also provide a novel perspective for the interpretation of previous results on conflict processing. For instance, a face-word Stroop study by Egner and Hirsch (2005) found that activity within the fusiform face area increased when faces were targets while there was no change in activity when faces were distractors, in line with the idea that no active inhibition is required for distractor processing once the brain has learned about the distractor (i.e., the face). What is more, Soutschek et al. (2015) manipulated the task difficulty in a picture-word interference task by varying the ratio of congruent to incongruent trials and found a modulatory effect of task difficulty expectations mainly on distractor but not on target processing. More precisely, the condition with a higher ratio of incongruent trials was characterized by lower activity within the visual word form area, which was responsible for the distractor processing. It seems that not only the learning of lower-level features such as spatial locations, but also learning of higher-level factors such as task difficulty, can facilitate distractor inhibition.

The neural mechanisms of learning about targets and distractors in the work by van Moorselaar and Slagter (2019) were explored based on the difference be- tween the last and the first trial of the visual search task. Complementarily, it would be revealing to investigate the learning process in its full extent and to ask whether ignoring a distractor over time is better described as a process of gradual accumulation or a sudden adjustment at some point in time. For the ERP data presented by van Moorselaar and Slagter (2019), it is tempting to speculate that the Pd amplitude might decrease rapidly after the first trial but only gradually thereafter, following the learning effect reflected in response speeding. Indeed, comparable ERP effects of learning have been reported. For instance, Cunillera et al. (2012) examined the P3 component induced by repetitive positive feedback in a Wisconsin card sorting task and found a marked decrease in P3 amplitude from the first to the second trial, and relatively subtle decreases for the following trials. Future studies may adopt the learning design and focus on the time course of neural activity underlying learning to shed light on the question of how attention allocation is modulated by learned expectation in a stepwise manner.

In summary, the work by van Moorselaar and Slagter (2019) dissociates learning effects on target facilitation (preparatory) and distractor suppression (reactive) in visual search and provides evidence for a predictive coding framework in which the processing of expected stimuli can be suppressed without topdown inhibition. These findings prompt interesting questions for future research. How are prestimulus target enhancement and poststimulus distractor suppression integrated to promote perceptual selection and decision making? How are target facilitation and distractor suppression organized in a scenario of crossmodal (e.g., audio-visual) stimulation? Can distractor suppression learned in one context generalize to other contexts?

\section{References}

Arnal LH, Giraud AL (2012) Cortical oscillations and sensory predictions. Trends Cogn Sci 16:390-398.

Baluch F, Itti L (2011) Mechanisms of top-down attention. Trends in neurosciences 34:210224.

Brouwer GJ, Heeger DJ (2009) Decoding and reconstructing color from responses in human visual cortex. J Neurosci 29:13992-14003.

Cunillera T, Fuentemilla L, Periañez J, MarcoPallarès J, Krämer UM, Càmara E, Münte TF, Rodríguez-Fornells A (2012) Brain oscillatory activity associated with task switching and feedback processing. Cogn Affect Behav Neurosci 12:16-33 
de Lange FP, Heilbron M, Kok P (2018) How do expectations shape perception? Trends Cogn Sci 22:764-779.

Egner T, Hirsch J (2005) Cognitive control mechanisms resolve conflict through cortical amplification of task-relevant information. Nat Neurosci 8:1784-1790.

Ferrante O, Patacca A, Di Caro V, Della Libera C, Santandrea E, Chelazzi L (2018) Altering spatial priority maps via statistical learning of target selection and distractor filtering. Cortex 102:67-95.

Foster JJ, Awh E (2019) The role of alpha oscillations in spatial attention: limited evidence for a suppression account. Curr Opin Psychol 29:34-40.

Friston K (2009) The free-energy principle: a rough guide to the brain? Trends Cogn Sci 13:293-301.

Grothe B (2018) How the barn owl computes auditory space. Trends in neurosciences 41 : 115-117.

Jensen O, Mazaheri A (2010) Shaping functional architecture by oscillatory alpha activity: gating by inhibition. Front Hum Neurosci 4:186.

Lavie N (2005) Distracted and confused?: selective attention under load. Trends Cogn Sci 9:75-82.

Noonan MP, Adamian N, Pike A, Printzlau F, Crittenden BM, Stokes MG (2016) Distinct mechanisms for distractor suppression and target facilitation. J Neurosci 36:1797-1807.

Noonan MP, Crittenden BM, Jensen O, Stokes MG (2018) Selective inhibition of distracting input. Behav Brain Res 355:36-47.

Payne L, Guillory S, Sekuler R (2013) Attentionmodulated alpha-band oscillations protect against intrusion of irrelevant information. J Cogn Neurosci 25:1463-1476.
Poch C, Valdivia M, Capilla A, Hinojosa JA, Campo P (2018) Suppression of no-longer relevant information in working memory: an alpha-power related mechanism? Biol Psychol 135:112-116.

Soutschek A, Stelzel C, Paschke L, Walter H, Schubert T (2015) Dissociable effects of motivation and expectancy on conflict processing: an fMRI study. J Cogn Neurosci 27:409423.

Summerfield C, Egner T (2009) Expectation (and attention) in visual cognition. Trends Cogn Sci 13:403-409.

van Moorselaar D, Slagter HA (2019) Learning what is irrelevant or relevant: expectations facilitate distractor inhibition and target facilitation through distinct neural mechanisms. J Neurosci 39:6953-6967. 